UV Radiation and Visible Light Induce hsp70 Gene Expression in the Antarctic Psychrophilic Ciliate Euplotes focardii

Lorenzo Fulgentini, Valerio Passini, Giuliano Colombetti, Cristina Miceli, Antonietta La Terza \& Roberto Marangoni

Microbial Ecology

ISSN 0095-3628

Microb Ecol

DOI 10.1007/s00248-015-0566-y
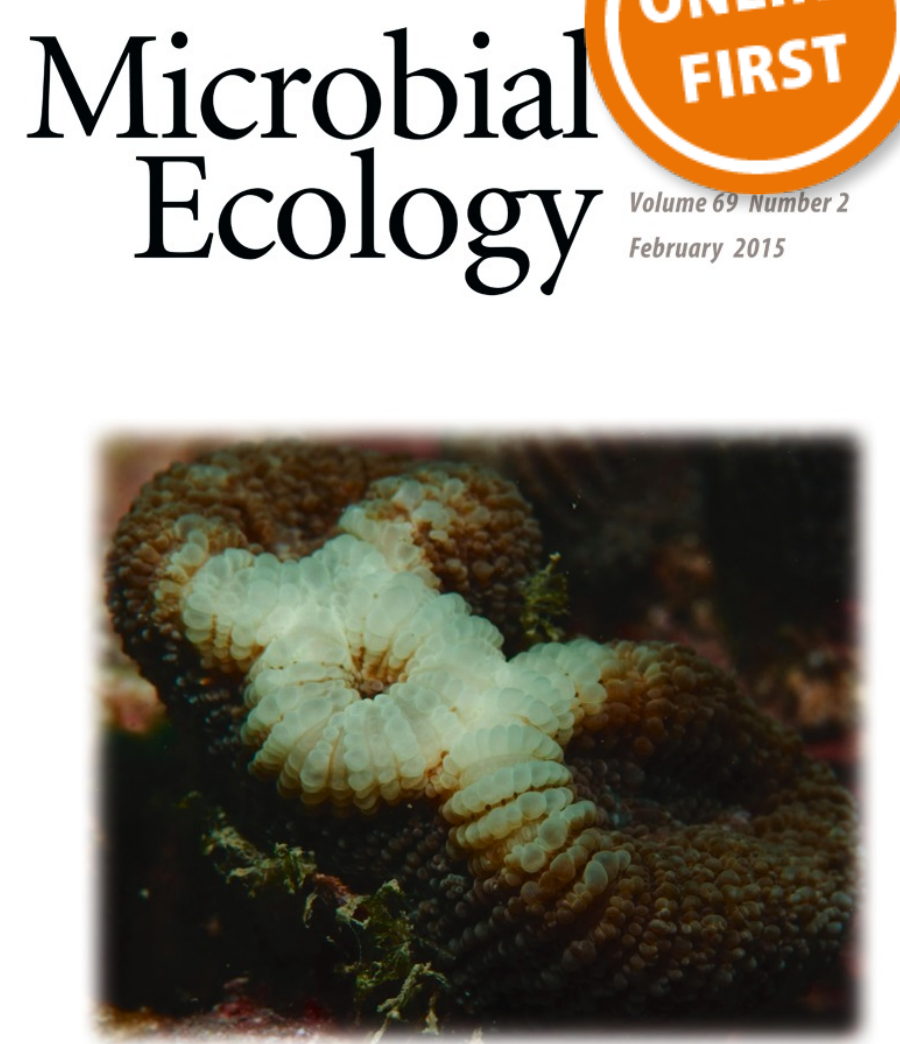
Your article is protected by copyright and all rights are held exclusively by Springer Science +Business Media New York. This e-offprint is for personal use only and shall not be selfarchived in electronic repositories. If you wish to self-archive your article, please use the accepted manuscript version for posting on your own website. You may further deposit the accepted manuscript version in any repository, provided it is only made publicly available 12 months after official publication or later and provided acknowledgement is given to the original source of publication and a link is inserted to the published article on Springer's website. The link must be accompanied by the following text: "The final publication is available at link.springer.com". 


\title{
UV Radiation and Visible Light Induce hsp 70 Gene Expression in the Antarctic Psychrophilic Ciliate Euplotes focardii
}

\author{
Lorenzo Fulgentini • Valerio Passini • Giuliano Colombetti • \\ Cristina Miceli • Antonietta La Terza • Roberto Marangoni
}

Received: 25 August 2014 / Accepted: 2 January 2015

(C) Springer Science+Business Media New York 2015

\begin{abstract}
The psychrophilic ciliate Euplotes focardii inhabits the shallow marine coastal sediments of Antarctica, where, over millions of years of evolution, it has reached a strict molecular adaptation to such a constant-temperature environment (about $-2{ }^{\circ} \mathrm{C}$ ). This long evolution at sub-zero temperatures has made $E$. focardii unable to respond to heat stress with the activation of its heat shock protein ( $h s p) 70$ genes. These genes can, however, be expressed in response to other stresses, like the oxidative one, thus indicating that the molecular adaptation has exclusively altered the heat stress signaling pathways, while it has preserved $h s p 70$ gene activation in response to other environmental stressors. Since radiative stress has proved to be affine to oxidative stress in several organisms, we investigated the capability of UV radiation to induce $h s p 70$ transcription. E. focardii cell cultures were exposed to several different irradiation regimes, ranging from visible only to a mixture of visible, UV-A and UV-B. The irradiation values of each spectral band have been set to be comparable with those recorded in a typical Antarctic spring. Using Northern blot analysis, we measured the expression level of $h s p 70 \mathrm{im}$ mediately after irradiation (0-h-labeled samples), $1 \mathrm{~h}$, and $2 \mathrm{~h}$
\end{abstract}

Antonietta La Terza and Roberto Marangoni are joint last authors.

L. Fulgentini $\cdot$ G. Colombetti $\cdot$ R. Marangoni

CNR-Biophysics Institute, via G. Moruzzi 1, 56100 Pisa, Italy

L. Fulgentini

CNR-National Institute of Optics, via G. Moruzzi 1, 56100 Pisa, Italy

V. Passini $\cdot$ C. Miceli $\cdot$ A. La Terza $(\square)$

School of Biosciences and Veterinary Medicine, University of Camerino, Via Gentile III da Varano, 62032 Camerino, MC, Italy

e-mail: antonietta.laterza@unicam.it

R. Marangoni $(\bowtie)$

Department of Biology, University of Pisa, via Derna 1,

56100 Pisa, Italy

e-mail: roberto.marangoni@unipi.it from the end of the irradiation. Surprisingly, our results showed that besides UV radiation, the visible light was also able to induce hsp70 expression in E. focardii. Moreover, spectrophotometric measurements have revealed no detectable endogenous pigments in E. focardii, making it difficult to propose a possible explanation for the visible light induction of its $h s p 70$ genes. Further research is needed to conclusively clarify this point.

Keywords Euplotes focardii $\cdot h s p 70 \cdot$ Gene regulation · Radiative stress · UV · Pigment · Visible light gene activation

\section{Introduction}

Over the last few years, the release of anthropogenic chlorofluorocarbons (CFCs) has caused remarkable changes in the chemistry of the atmosphere, especially regarding the ozone layer. Ozone $\left(\mathrm{O}_{3}\right)$ is the only component of the atmosphere capable of absorbing the UV-B spectral band (280-315 nm) of solar light. It is continuously destroyed by a process involving especially $\mathrm{Cl}$ radicals that derive from $\mathrm{CFC}$ photo-degradation in the stratosphere. Ozone depletion is more severe over the Antarctica, where ozone levels decline dramatically (typically $>50 \%$ ) during austral spring resulting in the formation of the so-called ozone hole [1]. These changes bring about variations in spectral distribution of solar radiation reaching the terrestrial surface, since they let the UV-B region of the solar spectrum pass through the stratospheric filter in a larger amount [2]. UV$\mathrm{B}$ radiation is the most biologically active, and its absorption by cells can cause severe damage: in fact, it may lead to the production of active oxygen species and free radicals capable of changing membrane and protein structure [3]. The absorption of UV-B by DNA, RNA and proteins causes mutagenesis and interferes with normal metabolic functioning. In plants, it 
can lead to the partial inhibition of photosynthesis. Long-term biological consequences include morphogenetic aberrations, impaired growth and restricted mobility [4].

The degree of UV sensitivity is related to the efficiency of repair mechanisms and to the existence of mitigating strategies, such as the presence of intracellular pigments capable of quenching and dissipating the excess energy before the formation of dangerous oxygen species $[4,5]$.

Even aquatic organisms are not completely safe; they are however protected by water which can partially screen electromagnetic radiation. In fact, even if light intensity decreases exponentially with the depth of the water column, biologically damaging levels of UV-B can penetrate to depths $>30 \mathrm{~m}$ (depending on sea state, turbidity and vertical mixing), affecting organisms in coastal regions and the upper photic zone of open oceans $[4,6,7]$. In this scenario, observations by many researchers show convincing evidence of UV-B damage to phytoplankton [8].

Concerning the Antarctic aquatic environment, the polar pack is an efficient barrier for light transmission (especially when covered with snow). It reflects $40-80 \%$ of incident radiation [4], and during austral spring, when the ozone hole reaches its climax, it is still quite extended. However, the plateau has completely ice-free local areas, called "polynya": in these regions, the radiation may reach major depths. For example, some evidence indicates that Antarctic zooplankton accumulates significant DNA damage during periods of increased UV-B flux [1, 9]. Moreover, the high concentrations of UV radiation protective mycosporine-like amino acids have been measured in sea-ice diatom samples [10].

Prolonged exposure to stress such as UV-B radiation may have negative consequences on the biochemical properties of proteins: in response to UV-B-induced protein impairment, cells may activate an evolutionary (universally) conserved cellular defence mechanism, known as the heat shock response, which involves the rapid synthesis of a set of general stress proteins known as "heat shock proteins" (HSPs). HSPs and in particular those belonging to the HSP70 family, behave as "molecular chaperones" assisting cells in chaperoning protein folding and assembly as well as in promoting damaged proteins degrading and thus contrasting the proteotoxic effects due to exposure to heat, UV radiation as well as to a wide variety of other environmental stressors [11-13].

Since its discovery by Ritossa [14], heat shock protein ( $h s p$ ) genes and their expression, mainly in response to heat and other different environmental stress stimuli, have been extensively analyzed in various organisms belonging to different kingdoms (from Prokaryotes to Eukaryotes). Quite recently, $h s p$ genes (and their expression) were also analyzed in organisms, living in Antarctica coastal seawater, a habitat characterized by an extremely cold $\left(-2{ }^{\circ} \mathrm{C}\right)$ and constant temperature (for a review, see [15]).
With regard to the conservation of the heat shock response in evolution, it is interesting to note that at least three species lacking an inducible heat shock response have been found; this lack might be due to evolutionary adaptation to extremely stable temperatures (firmly in the range of $-2{ }^{\circ} \mathrm{C}$ ) [16]. These species are Hydra oligactis, a freshwater cnidarian in which this phenomenon has been described for the first time $[17,18]$ and two Antarctic species: the fish Trematomus bernacchii [19] and the protozoan ciliate Euplotes focardii [20].

E. focardii is an hypotrichous bottom-dwelling ciliate, endemic in Antarctic coastal seawater and among the various Euplotes species of Antarctic origin up to now described, is considered to be one of the most interesting to study because its adaptation to the cold does seem to be more remote than that of the other Antarctic species [21]. Evidence of this marked psychrophilic (cold-loving) behaviour comes from the observation that this species shows optimal survival and growth rates at $4-5{ }^{\circ} \mathrm{C}$ and cannot survive at a temperature above $10^{\circ} \mathrm{C}$ [21]. Moreover, and with respect to the molecular adaptive strategies so far investigated, the $E$. focardii tubulins have evolved numerous peculiar post-translational modifications, to prevent microtubules de-polymerization at sub-zero temperatures [22, 23], and its $h s p 70$ genes have lost the capacity to be effectively transcriptionally activated in response to environmental thermal shock although these genes have preserved their capacity to be induced in response to other non-thermal (chemical and oxidative) shock [24].

Thus, E. focardii in view of its strict psychrophilic behaviour represents an intriguing bio-model to explore due to its peculiar molecular adaptive strategies in extreme (cold) environments.

On a recent paper, Di Giuseppe et al. [25] investigated the sensitivity of different Euplotes species of polar (E. focardii and Euplotes nobilii) and non-polar (Euplotes quinquecarinatus and Euplotes raikovi) origin to UV radiations. By measuring the cell viability of the various Euplotes species under different regimes of UV-B and UV-A exposures, these authors reported that the polar species are able to face UV radiation more efficiently than nonpolar ones. In particular, E. focardii showed the strongest resistance to UV-B radiation.

Given these premises, the present work was aimed at starting an investigation of the molecular basis of UV resistance in E. focardii. Regarding this, we examined the capacity of $E$. focardii to withstand UV radiations as well as other combinations of spectral components, with the activation of its $h s p 70$ genes. Northern blot analysis of total RNA extracted from $E$. focardii exposed to UV-A and UV-B radiations revealed a consistent induction of $h s p 70$ gene expression. Surprisingly, our analysis showed that besides UV radiations, visible light by itself was also able to appreciably induce E. focardii hsp 70 expression. Since spectrophotometric measurements have revealed no detectable endogenous pigments 
in E. focardii; at the moment, it is difficult to propose a possible/plausible signaling pathway for the visible light induction of $h s p 70$ expression, and further research is needed to clarify this phenomena.

\section{Materials and Methods}

\section{Cell Cultures}

The results described in the present paper were obtained from experiments carried out on culture of $E$. focardii cells belonging to strain 95 (the same strain used by Di Giuseppe et al. [25] to analyze UV radiation effects in various Euplotes species). This strain represents type-species material chosen from a number of wild-type strains isolated from sediment and seawater samples collected from the coastal waters of Terra Nova Bay, Antarctica, where it is located in the Italian base "Mario Zucchelli" (S 74 $42^{\prime} / \mathrm{E} 164^{\circ} 06^{\prime}$ ). In particular, this strain was collected during the $\mathrm{X}$ expedition to Antarctica that was held in 1995 (http://www.pnra.it).

The strain was grown in artificial sea water (33\%, $\mathrm{pH} 8.1-$ 8.2) in a cold room at a temperature of $4{ }^{\circ} \mathrm{C}$ as in Di Giuseppe et al. [25]. They were fed with the green algae Dunaliella tertiolecta. Stress conditions (UV and visible light exposures) were applied to cells after about 1 week of vegetative proliferation in the presence of food. Two days before irradiation, the cells were induced to uniformly arrest their division cycle in the G1-G0 (stationary) phase by depleting the food as described in La Terza et al. [20].

\section{Irradiation Protocols}

Immediately before applying the irradiation protocols, control (non-irradiated) and experimental (irradiated) cell samples were concentrated by mild centrifugation for $5 \mathrm{~min}$ at $3500 \mathrm{rpm}(2000 \mathrm{rcf})$ at $4{ }^{\circ} \mathrm{C}$ and re-suspended in seawater at the density of approximately $2-3000$ cells per millilitre, in beakers of $40 \mathrm{ml}$ (i.e. a total amount of about 12,000 cells per sample).

All the irradiation protocols were carried out in a temperature-controlled chamber $\left(4^{\circ} \mathrm{C}\right)$. Cell cultures of E. focardii were covered with one suitable filter (chosen among the following: Schott WG280 cut-off, GG400 cut-off and UG1 broadband) in order to expose them to the following three different spectral compositions (each experimental condition is named after the filter used):

- GG400 samples: the samples covered by GG400 filter received exclusively visible light. Culture samples were exposed for 90 min using three UV-B (Philips TL $20 \mathrm{~W} /$ 12 RS), one UV-A (Philips TLK $40 \mathrm{~W} / 05$ ) and two visible (Philips TLD $18 \mathrm{~W} / 54$ ) fluorescent tubes.

- WG280 samples: they received a combination of UV-B + UV-A + visible radiations. Culture samples were exposed for 90 min using the same combinations of fluorescent tubes used for the GG400 samples.

- UG1 samples: they received exclusively UV-B + UV-A radiations, but not the visible light component. Culture samples were exposed for $120 \mathrm{~min}$ using five UV-B (Philips TL 20 W/12 RS) fluorescent tubes.

It should be noted that differences in the exposure times and illumination set-up between UG1 sample and the GG400 and WG280 samples, is due to the need to treat the E. focardii cultures with the same total dose of UV band (UV-A + UV-B) and to maintain its value similar to that of an Antarctic spring morning (as recorded by "NSF Polar UV Monitoring Network", see [26]).

Figure 1 shows the spectral distributions and the irradiance values of the irradiation reaching each sample. Spectral irradiance was measured by means of a calibrated radiometric spectrograph (Oriel Instaspec IV). Table 1 shows UV-A, $\mathrm{UV}-\mathrm{B}$ and visible radiation irradiances (in $\mathrm{W} \mathrm{m}^{-2}$ ) and total

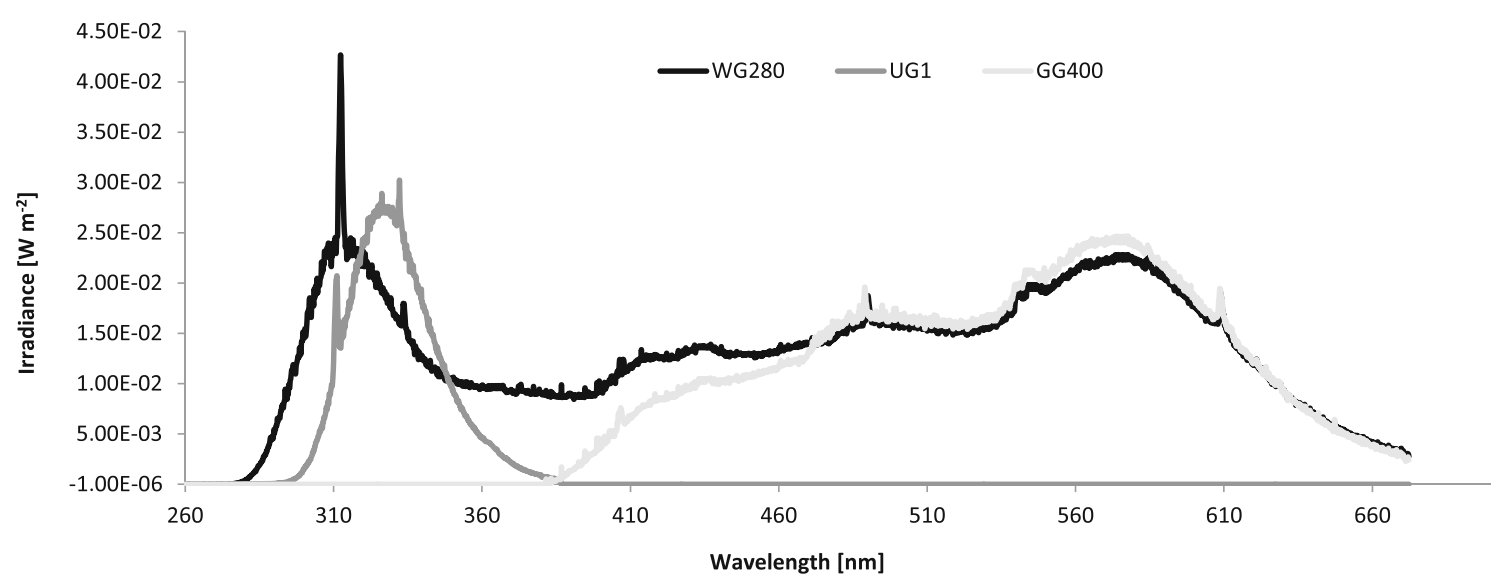

Fig. 1 UV and visible spectral distributions for each filter used in the experiment. The different curves represent the spectral composition of the polychromatic irradiation received by each sample. In the abscissa, the wavelength (in $\mathrm{nm}$ ); in the ordinate, the irradiance value (in $\mathrm{W} \mathrm{m}^{-2}$ ) 
Table 1 Irradiance values in $\left(\mathrm{W} \mathrm{m}^{-2}\right)$ (in italic) and total exposure dose in $\left(\mathrm{J} \mathrm{m}^{-2}\right)$ (plain text) for each sample in the three main spectral bands (UV-B, UV-A and visible)

\begin{tabular}{llll}
\hline & UG1 & WG480 & GG400 \\
\hline UV-B & 0.2 & 0.46 & 0.00 \\
& 1100.00 & 2500.00 & 0.00 \\
UV-A & 1.28 & 1.07 & 0.03 \\
& 6900.00 & 5800.00 & 150.00 \\
Vis. & 0.00 & 4.86 & 4.86 \\
& 0.00 & $26,250.00$ & $26,250.00$ \\
\hline
\end{tabular}

doses (in $\mathrm{J} \mathrm{m}^{-2}$ ) received by each sample. Exposure times were set in order to maintain the total exposure dose in the UV-B band below the threshold of $11 \mathrm{~kJ} \mathrm{~m}^{-2}$ that, in agreement with Di Giuseppe et al. [25], were able to ensure the survival of at least $60 \%$ of the cells. Experiments were repeated three times with four replicates for each of the assayed experimental condition.

\section{RNA Extraction}

Total RNA was extracted from control and irradiated E. focardii cell cultures using TRIzol reagent (Life Technologies) according to the manufacturer's protocol. E. focardii cells were harvested by mild centrifugation, and RNA was extracted just after the end of each planned irradiation period ( 0 -h sample) and after $1 \mathrm{~h}$ (1-h sample) and $2 \mathrm{~h}$ (2-h sample).

\section{Northern Blotting Analysis}

Equal amounts of total RNA preparations $(40 \mu \mathrm{g} / \mathrm{lane})$ were analyzed by Northern blottings after electrophoresis on $1.2 \%$ formaldehyde denaturing agarose gel, according to standard procedures [27]. Hybridization was carried out with two different types of 32P-labeled probes [28] represented by $E$. focardii: hsp70 gene sequences specific for the protein catalytic domain [24] and E. focardii: $17 \mathrm{~S}$ ribosomal DNA (rDNA) gene sequences. Blotted membranes (Hybond-N, Amersham Biosciences) were pre-hybridized for $1 \mathrm{~h}$ in $5 \times$ Denhardt's solution, $0.5 \%$ sodium dodecyl sulfate (SDS) and $10 \mathrm{mM}$ EDTA. Probes were boiled for 5 min and snap cooled on ice before being added to the hybridization solution containing $6 \times$ saline-sodium citrate (SSC), $5 \times$ Denhardt's solution, $0.1 \%$ SDS and $100 \mu \mathrm{g} / \mathrm{ml}$ of denatured tRNA. All hybridizations were carried out overnight at $65{ }^{\circ} \mathrm{C}$ with a constant agitation, and membranes were washed twice with $0.5 \times \mathrm{SSC}$ containing $0.1 \%$ SDS, at the hybridization temperature for $1 \mathrm{~h}$, then dried and exposed for autoradiography. Equal loading of RNA samples was evaluated by re-probing the filters firstly hybridized with the hsp 70 probes, with a homologous $17 \mathrm{~S}$ rDNA probe. Both probes were used at the equal number of counts per minute (106). Membranes were re-probed after having been incubated with a boiling solution of $0.1 \%$ SDS and allowed to cool to room temperatures. The intensity of hybridization bands was measured using an Imagining Densitometer GS-670 (Bio-Rad).

\section{Spectrophotometric Pigments Assay}

Cells were dissolved in acetone and centrifuged, and the supernatant was dried under a vacuum, re-suspended in ethanol and analyzed by means of a Jasco V550 UV/VIS spectrophotometer. The same procedure was applied to $D$. tertiolecta for the extraction of its pigments.

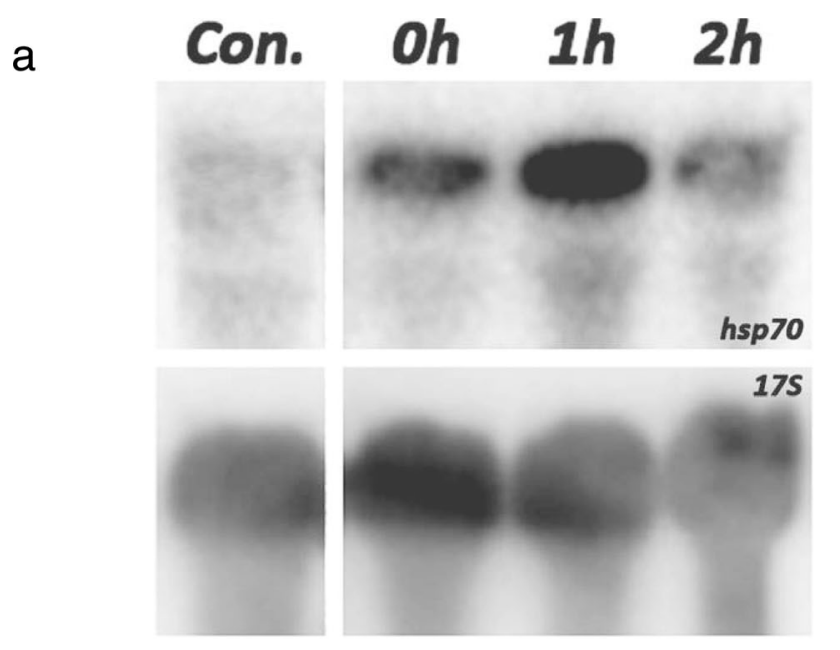

b

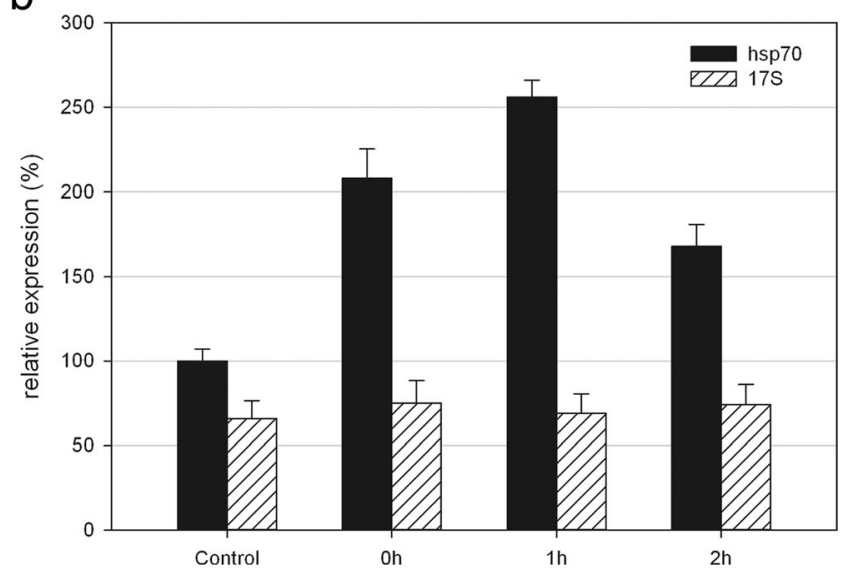

Fig. 2 a Northern blot analysis of total RNA preparations from cells covered with UG1 (UV-B + UV-A) filter and extracted 0,1 and $2 \mathrm{~h}$ after the end of the irradiation. An equal amount $(40 \mu \mathrm{g})$ of each preparation was fractionated by electrophoresis, blotted and assayed, first, with the E. focardii hsp 70 probe (top) and, then, with the Euplotes crassus $17 S$ rDNA probe (bottom). b The graph shows the relative intensities (in percentage) for both $h s p 70$ and $17 S$ probes, normalized by taking the control expression level of $h s p 70$ as $100 \%$. Error bars refer to the standard error of the mean 


\section{Results and Discussion}

To assess the capacity of the psychrophilic Antarctic ciliates E. focardii to raise the expression of its $h s p 70$ genes in response to UV radiation as well as to other spectral compositions, we exposed equal cell samples to the following different irradiation regimes: 1) UV-A and UV-B radiations (UG1 samples); 2) a mixture of visible, UV-A and UV-B radiations (WG280 samples); and 3) visible light alone, without the UV-A and UV-B components (GG400 samples). For all the spectral compositions, the irradiation values of each spectral band were regulated in order to be comparable with those recorded in a typical Antarctic spring. From this point of view, our irradiation protocols differed from those used in the study of Di Giuseppe et al. [25]. In fact, these Authors used irradiation values similar

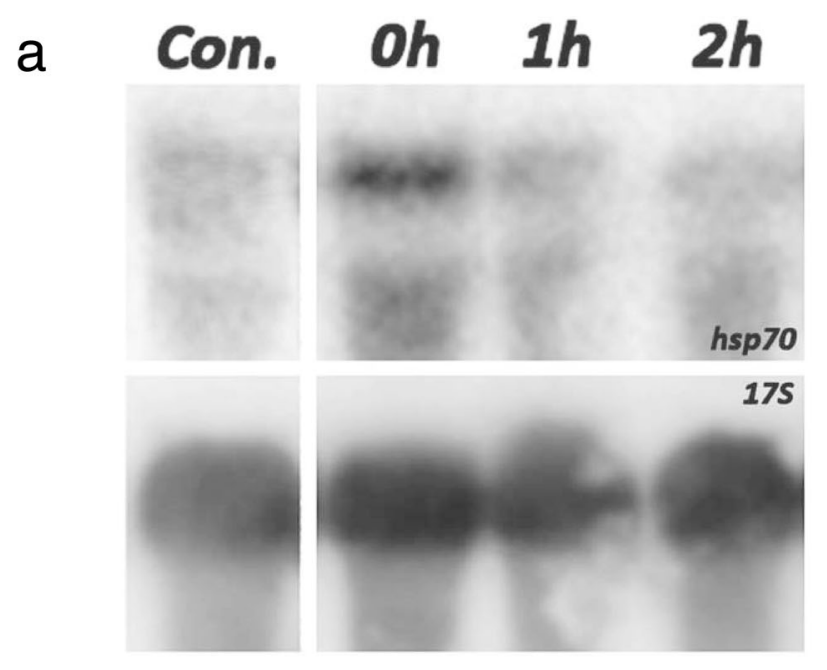

b

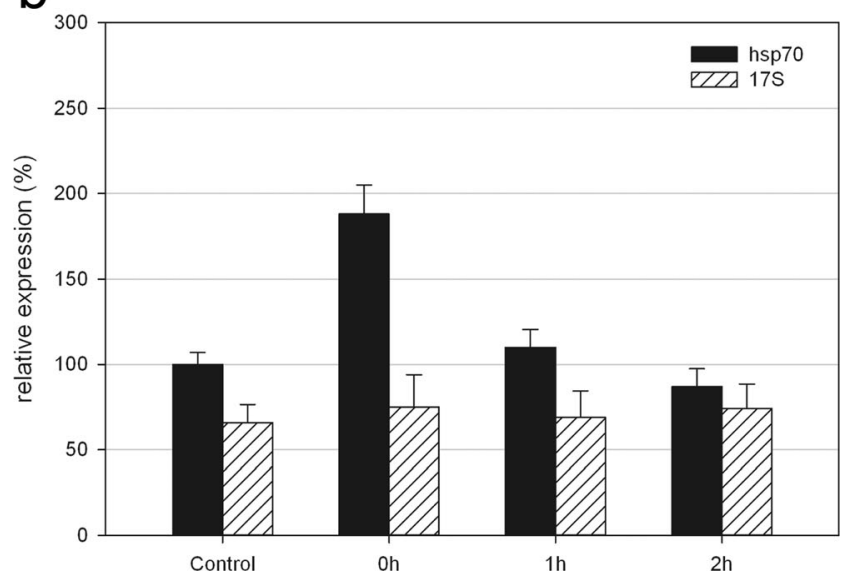

Fig. 3 a Northern blot analysis of total RNA preparations from cells covered with WG280 (UV-B + UV-A + visible) filter and extracted 0, 1 and $2 \mathrm{~h}$ after the end of the irradiation. An equal amount $(40 \mu \mathrm{g})$ of each preparation was fractionated by electrophoresis, blotted and assayed, first, with the E. focardii hsp 70 probe (top) and, then, with the Euplotes crassus $17 S$ rDNA probe (bottom). b The graph shows the relative intensities (in percentage) for both $h s p 70$ and $17 S$ probes, normalized by taking the control expression level of $h s p 70$ as $100 \%$. Error bars refer to the standard error of the mean to those recorded at noon in a mid-latitude region in summertime. Therefore, we decided not to use the same high irradiance levels used by Di Giuseppe et al. [25] since they were too high to have an ecological relevance for $E$. focardii. In fact, those ciliates would never be exposed to those levels of UV-B, even in the case of a complete disruption of the Antarctic stratospheric ozone layer. Instead, we chose to set the irradiances to values comparable with those recorded during Antarctic spring, keeping the total UV-B dose supplied to the cell samples below the threshold of $11 \mathrm{~kJ} \mathrm{~m}^{-2}$, which, according to the work of Di Giuseppe et al., corresponds to a mortality of about $40 \%$, thus allowing us to analyze the sub-lethal effects of UV radiation at the molecular level.

The expression levels of E. focardii hsp 70 transcripts following the different irradiation regimes were measured by

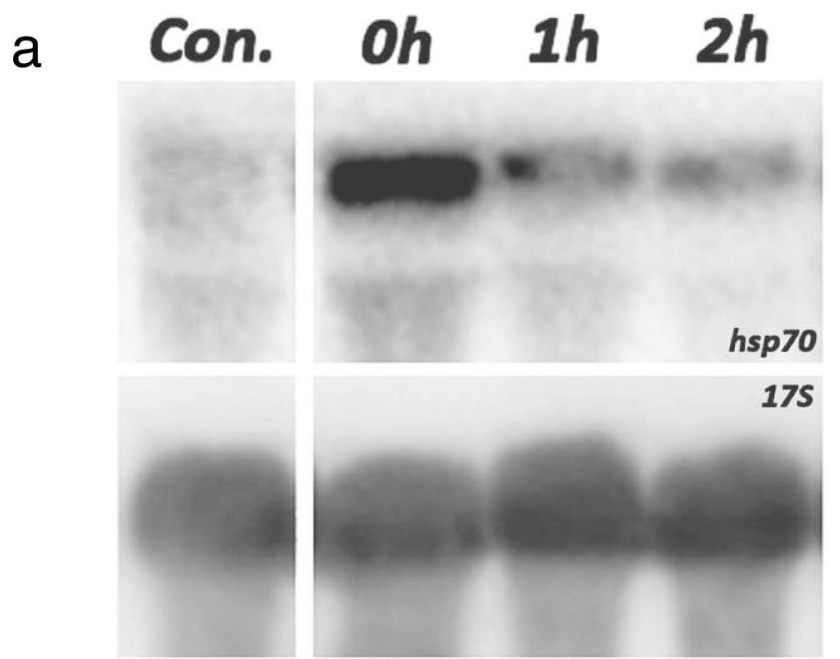

b

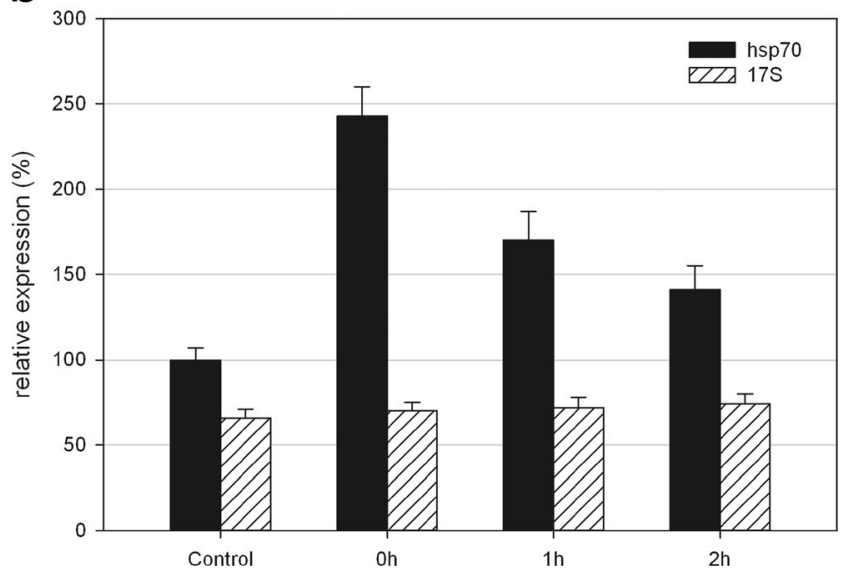

Fig. 4 a Northern blot analysis of total RNA preparations from cells covered with GG400 (visible light) filter and extracted 0,1 and $2 \mathrm{~h}$ after the end of the irradiation. An equal amount $(40 \mu \mathrm{g})$ of each preparation was fractionated by electrophoresis, blotted and assayed, first, with the E. focardii hsp 70 probe (top) and, then, with the Euplotes crassus $17 S$ rDNA probe (bottom). b The graph shows the relative intensities (in percentage) for both $h s p 70$ and $17 S$ probes, normalized by taking the control expression level of $h s p 70$ as $100 \%$. Error bars refer to the standard error of the mean 
Northern blot analysis using as probes the E. focardii Hsp70 ATP binding domain [24]. To evaluate the effects of the different irradiation regimes on the kinetics of hsp70 gene expression in E. focardii, samples of total RNA were extracted from irradiated cells immediately after the end of the irradiation period (0-h-labeled sample), $1 \mathrm{~h}$ (1-h sample) and $2 \mathrm{~h}$ (2-h sample) later.

As shown in Fig. 2a, the E. focardii cells exposed to UV-B + UV-A radiations (UG1 sample) as described in "Materials and Methods", produced an intense 2.1-kbp band, i.e. the molecular size expected for $h s p 70 \mathrm{mRNA}$, immediately after the end of the irradiation period ( 0 -h sample). The hybridization signals peaked $1 \mathrm{~h}$ later (reaching about $256 \%$ of the control sample expression level, see Fig. 2b) and showed a significant decrease over the following $2 \mathrm{~h}$ at the end of irradiation. To exclude any qualitative-quantitative variations between RNA samples, the blots were stripped and rehybridized with the E. focardii $17 \mathrm{~S}$ rDNA probe, and Fig. 2a shows equivalent hybridization signals.

Next, E. focardii cells were exposed to a combination of UV-B + UV-A + visible radiations (WG280 sample). Also, in this case, the $h s p 70$ gene showed a prompt up-regulation in the 0 -h samples (about $188 \%$ with respect to the expression level of the control sample, see Fig. 3b) and a gradual decrease of the transcript levels over the following $2 \mathrm{~h}$ (1- and 2-h samples, see Fig. 3b).

Surprisingly and as shown in Fig. 4, visible radiations by itself were also able to up-regulate $h s p 70$ gene expression in E. focardii cells. Exposure to visible light produced an $h s p 70$ transcription profile similar to that shown by the WG280 sample, described above (compare Fig. 3b with Fig. 4b).

Remarkably, from a behavioural point of view, the visible light-exposed cells were absolutely indistinguishable from control (non-irradiated) cells: they in fact, maintain the same morphology and motility, while in the other two samples comprising the UV bands, the cells showed an altered morphology with the appearance of anomalous big vacuoles inside the cytoplasm and a reduced motility (data not shown).

Our data clearly shows that Antarctic psychrophilic ciliate E. focardii is capable of reacting to radiative stress triggered by different spectral components (UV-A, UV-B or even visible light alone), eliciting the transcriptional activation of its $h s p 70$ genes. Apart from the UV-mediated $h s p 70$ response which could be somehow expected for this Antarctic microorganism, the most amazing results were represented by the capacity of the visible light to trigger $h s p 70$ expression.

How can the $h s p 70$ gene expression be activated in response to radiative stresses, as well as to visible light? Previous research has showed that $E$. focardii is no longer capable of responding to environmental thermal shocks with an effective activation of a transcriptional response of its $h s p 70$ gene, although this gene still preserves its capacity to be activated in response to chemical as well as oxidative stressors [20]. Some hints to explain this eccentric transcriptional behaviour may reside in the peculiar organization of the 5 ' regulatory regions of the $E$. focardii hsp 70 gene [29]. The 5 ' regulatory region of $h s p 70$ gene in E. focardii includes two sequence motifs known to be specific for stress-inducible genes: the first regulation complex is identified by four motifs which are distinctive for their "heat shock promoter elements" (HSE) [30] that are targets of a specific class of transcriptional activators known as "heat shock factors" (HSF) [16]; the second complex is identified by another four motifs which are distinctive for "stress response elements" (StREs) [31, 32]. Thus, at least in principle, this gene can rely on two distinct cis-acting mechanisms to produce a stress-induced response, with HSE being

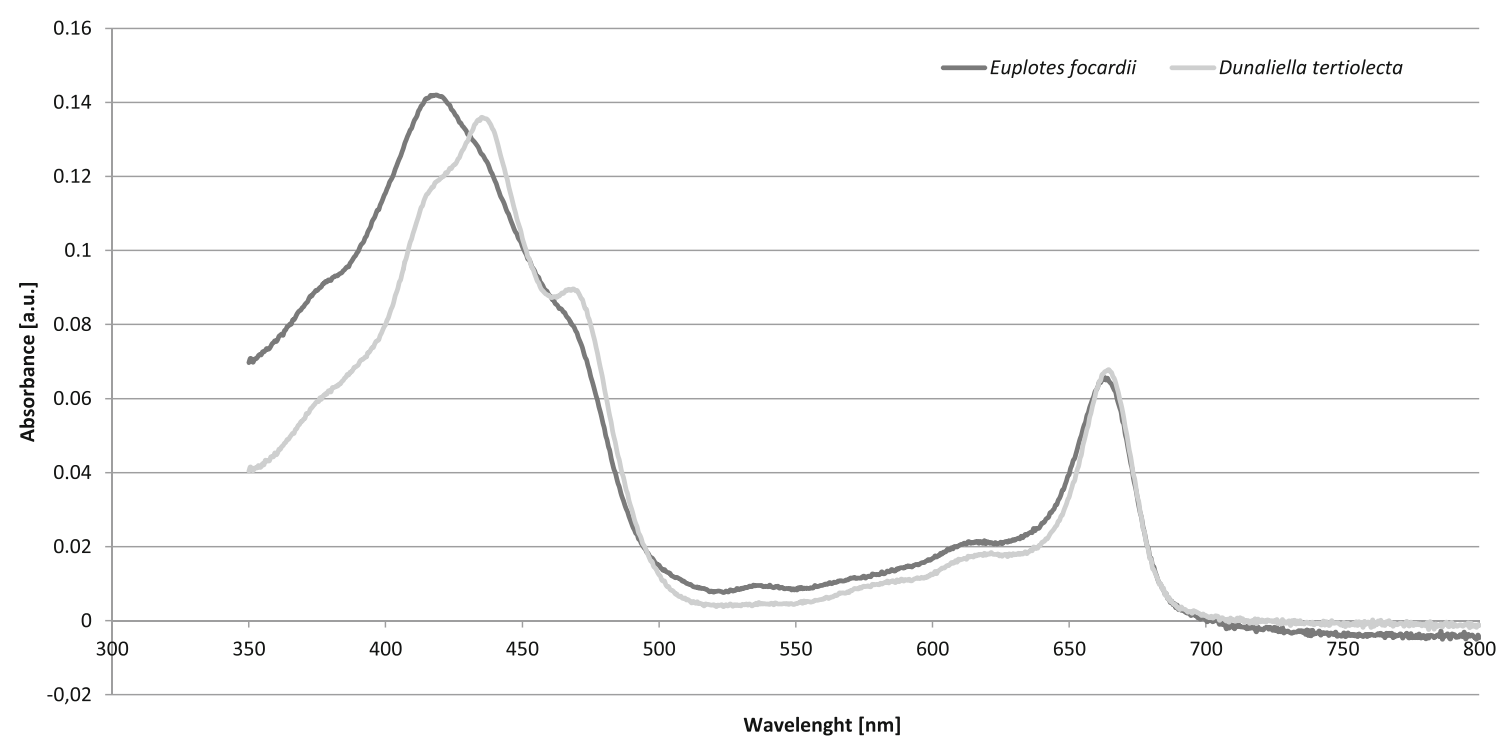

Fig. 5 Relatively normalized absorption spectra of Euplotes focardii and Dunaliella tertiolecta extracts. In the abscissa, the wavelength (in nm) of the incident light, in the ordinate the optical absorbance (in arbitrary unities) 
more specific to respond to a stress of a thermal nature and StRE being responsible for the response to a broader range of stress, such as oxidative and chemical ones [33, 34].

StREs could probably be the regulative elements involved in the response mediated by ultraviolet radiation, as UV-Bs are the most biologically damaging wavelengths, leading to the establishment of a strong oxidative environment inside the cell, with the production of active oxygen species and free radicals.

More puzzling is the activation of $h s p 70$ gene transcription after irradiation with visible light only; this would need the activation of one or more endogenous chromophores, which act as photosensitizers, capable of directly or indirectly damaging cellular components via excitation at wavelengths at which DNA does not absorb. Among these photosensitizers, we can recall methylene blue, acridine orange, riboflavin and porphyrins $[35,36]$. Even $\beta$-carotene or one of its decomposition products can itself act as a photosensitizer [37]. Nevertheless, the presence of such molecules in E. focardii has never been proven, and it is reasonable to think that they are present in a very small quantity in the digestive vacuoles, as sub-products of the digestion of the $D$. tertiolecta algae.

The activation of the $h s p 70$ gene transcription after irradiation with visible light only has been found in other organisms, such as in Chlamydomonas, where the responses to heat shock and light are quite similar. In particular, these authors showed that the $h s p 70$ gene expression in response to visible light was mediated through a heat shock-independent signal pathway involving a lightactivated factor [38]. Chlamydomonas, cells incubated in the dark and then shifted to light intensities that caused photoinhibition, photosystem II were less damaged and recovered faster when, prior to light stress, the cultures were pre-exposed to dim light for $60 \mathrm{~min}$. During this pre-incubation time, the $h s p 70$ genes were induced [39]. Experimental evidence showed that the chlorophyll precursors Mg-protoporphyrin IX or its dimethyl ester play crucial roles as intermediates in the signaling pathway by which light activates the expression of the nuclear heat shock genes [40], once these plastidic compounds get access to the cytoplasm/nucleus [41].

E. focardii could then possess some kind of photosensitizers, which can absorb visible light and trigger the response, even if this ciliate appears completely rid of pigments. To deeper investigate this point, after starving the cells for several days (up to 21 days), we tried to extract this putative pigment. Results (Fig. 5) show that chlorophyll deriving from the algae this ciliate feeds on is the only pigment that seems to be present inside $E$. focardii's cells, even after several days of starvation. It is puzzling, anyway, to imagine that these pigments may have an active signaling role, considering that they are already in the digestive vacuoles, and thus are inclined towards the degradation pathway.
The apparent absence of any endogenous pigment and the complete absence of a synthesis pathway for the chlorophyll (as E. focardii is not a photosynthetic organism) depict a very puzzling situation: it is hard to suggest a signaling pathway for the visible light-induced transcription of $h s p 70$. This situation recalls that of the histophagous ciliate, Ophryoglena flava, that shows a strong phototactic response to visible light, even if it is apparently free of pigments and fed on non-photosynthetic tissues (in particular, beef spleen, see [42]). Maybe in both these ciliates, pigments are contained in such a small quantity, to be undetectable by our instruments. Further investigations are required to address this point. Overall, this finding aims to provide a better understanding of molecular responses upon UV hsp70 induction as well as to unveil other intriguing aspects (visible light induction) of the adaptive strategies adopted by $E$. focardii to survive in the Antarctica.

\section{References}

1. Malloy KD, Holman MA, Mitchell D, Detrich HW III (1997) Solar UVB-induced DNA damage and photoenzymatic DNA repair in Antarctic zooplankton. Proc Natl Acad Sci U S A 94(4):1258-1263

2. Acevedo J, Nolan C (eds) (1993) Environmental UV radiation: causes - effects - consequences. Commission of the European Communities, Directorate-General XII for Science, Research and Development, Environment Programme, Brussels

3. Caldwell MM, Björn LO, Bornman JF, Flint SD, Kulandaivelu G, Teramura AH, Tevini M (1998) Effects of increased solar ultraviolet radiation on terrestrial ecosystems. J Photochem Photobiol B Biol 46: 40-52

4. Kennedy AD (1995) Antarctic terrestrial ecosystem response to global environmental change. Annu Rev Ecol Syst 26:683-704

5. Karsten U (2008) Defense strategies of algae and cyanobacteria against solar ultraviolet radiation. In: Amsler DC (ed) Algal chemical ecology. Springer, Berlin, pp 273-296

6. Agustí A (2008) The impact of increased ultraviolet radiation on the polar oceans. In: Duarte CM (ed) Impacts of global warming on polar ecosystems. Fundación BBVA, Bilbao, pp 25-45

7. Llabrés M, Agustí S (2010) Effects of ultraviolet radiation on growth, cell death and the standing stock of Antarctic phytoplankton. Aquat Microb Ecol 59:151-160

8. Häder DP, Helbling EW, Williamson CE, Worrest RC (2011) Effects of UV radiation on aquatic ecosystems and interactions with climate change. Photochem Photobiol Sci 10(2):242-60

9. Hansson L-A, Hylander S (2009) Effects of ultraviolet radiation on pigmentation, photoenzymatic repair, behavior, and community ecology of zooplankton. Photochem Photobiol Sci 8:1266-1275

10. Thomas DN, Dieckmann GS (2002) Antarctic sea ice- a habitat for extremophiles. Science 295:641-644

11. Whitley D, Goldberg SP, Jordan WD (1999) Heat shock proteins: a review of the molecular chaperones. J Vasc Surg 29(4):748-751

12. Sarkar S, Singh DM, Yadav R, Arunkumar KP, Pittman GW (2011) Heat shock proteins: molecules with assorted functions. Front Biol 6: 312-327

13. La Terza A, Barchetta S, Buonanno F, Ballarini P, Miceli C (2008) The protozoan ciliate Tetrahymena thermophila as biosensor of sublethal levels of toxicants in the soil. Fresenius Environ Bull 17:1144-1150 
14. Ritossa F (1962) A new puffing pattern induced by temperature shock and DNP in drosophila. Experientia 18:571-573

15. Clark MS, Peck LS (2009) HSP70 heat shock proteins and environmental stress in Antarctic marine organisms: a mini-review. Mar Genomics 2:11-18

16. Pirkkala L, Nykänen P, Sistonen L (2001) Roles of the heat shock transcription factors in regulation of the heat shock response and beyond. FASEB J 15:1118-1131

17. Bosch TCG, Krylow SM, Bode HR, Steele RE (1988) Thermotolerance and synthesis of heat shock proteins: these responses are present in Hydra attenuata but absent in Hydra oligactis. Proc Natl Acad Sci U S A 85:7927-7931

18. Brennecke T, Gellner K, Bosch TC (1998) The lack of a stress response in Hydra oligactis is due to reduced hsp70 mRNA stability. Eur J Biochem 255:703-709

19. Hofmann GE, Buckley BA, Airaksinen S, Keen JE, Somero GN (2000) Heat-shock protein expression is absent in the Antarctic fish Trematomus bernacchii (family Nototheniidae). J Exp Biol 203: 2331-2339

20. La Terza A, Papa G, Miceli G, Luporini P (2001) Divergence between two Antarctic species of the ciliate Euplotes, E. focardii and E. nobilii, in the expression of heat shock protein 70 genes. Mol Ecol 10:1061-1067

21. Valbonesi A, Luporini P (1993) Biology of Euplotes focardii, an Antarctic ciliate. Polar Biol 13:489-493

22. Pucciarelli S, Ballarini P, Miceli C (1997) Cold-adapted microtubules: characterization of tubulin posttranslational modifications in the antarctic ciliate Euplotes focardii. Cell Motil Cytoskeleton 38:329-340

23. Pucciarelli S, Miceli C (2002) Characterization of the cold-adapted atubulin from the psychrophilic ciliate Euplotes focardii. Extremophiles 6:385-389

24. La Terza A, Miceli C, Luporini P (2004) The gene for the heat-shock protein 70 of Euplotes focardii, an Antarctic psychrophilic ciliate. Antarct Sci 16(1):23-28

25. Di Giuseppe G, Cervia D, Vallesi A (2012) Divergences in the responses to ultraviolet radiation between polar and non-polar ciliated protozoa. Microb Ecol 63:634-638

26. Booth CR, Lucas TB, Morrow JH, Weiler CS, Penhale PA (1994) The United States National Science Foundation's Polar Network for monitoring ultraviolet radiation. In: Weiler CS, Penhale PA (eds) Ultraviolet radiation in Antarctica: measurements and biological effects, Antarct. Res. Ser. vol. 62. AGU, Washington, pp 17-37

27. Sambrook J, Fritsch EF, Maniatis T (eds) (1989) Molecular cloning-a laboratory manual, 2nd edn. New York, Cold Spring Habour Laboratory Press

28. Feinberg AP, Vogelstein B (1984) A technique for radiolabeling DNA restriction endonuclease fragments to high specific activity. Anal Biochem 137(1):266-267
29. La Terza A, Miceli C, Luporini P (2007) Adaptive evolution of the heat-shock response in the Antarctic psychrophilic ciliate, Euplotes focardii: hints from a comparative determination of the $h s p 70$ gene structure. Antarct Sci 16(2):239-244

30. Fernandes M, Xiao H, Lis JT (1994) Fine structure analyses of the Drosophila and Saccharomyces heat shock factor-heat shock element interactions. Nucl Acids Res 22:167-173

31. Kobayashi N, McEntee K (1993) Identification of cis and trans components of a novel heat shock stress regulatory pathway in Saccharomyces cerevisiae. Mol Cell Biol 13:248-256

32. Ruis H, Schüller C (1995) Stress signaling in yeast. BioEssays 17: 959-965

33. Feder ME, Hofmann GE (1999) Heat-shock proteins molecular chaperones, and the stress response: evolutionary and ecological physiology. Ann Rev Physiol 61:243-282

34. Estruch F (2000) Stress-controlled transcription factors stressinduced genes and stress tolerance in budding yeast. FEMS Microb Rev 24:469-486

35. Kielbassa C, Roza L, Epe B (1997) Wavelength dependence of oxidative DNA damage induced by UV and visible light. Carcinogenesis 18:811-816

36. Epe B, Pflaum M, Boiteux S (1993) DNA damage induced by photosensitizers in cellular and cell-free systems. Mutat Res 299:135145

37. Pflaum M, Kielbassa C, Garmyn M, Epe B (1998) Oxidative DNA damage induced by visible light in mammalian cells: extent, inhibition by antioxidants and genotoxic effects. Mutat Res/DNA Repair 408(2):137-146

38. Kropat J, Gromoff ED, Müller FW, Beck CF (1995) Heat shock and light activation of a Chlamydomonas HSP70 gene are mediated by independent regulatory pathways. Mol Gen Genet 248:727-734

39. Kropat J, Oster U, Rüdiger W, Beck CF (1997) Chlorophyll precursors are signals of chloroplast origin involved in light induction of nuclear heat-shock genes. Proc Natl Acad Sci U S A 94: 14168-14172

40. Kropat J, Beck CF (1998) Characterization of photoreceptor and signaling pathway for light induction of the Chlamydomonas heatshock gene HSP70A. Photochem Photobiol 68:414-419

41. Kropat J, Oster U, Rüdiger W, Beck CF (2000) Chloroplast signalling in the light induction of nuclear HSP70 genes requires the accumulation of chlorophyll precursors and their accessibility to cytoplasm/ nucleus. Plant J 24:523-531

42. Cadetti L, Marroni F, Marangoni R, Kuhlmann H-W, Gioffre D, Colombetti G (2004) Phototaxis in the ciliated protozoan Ophryoglena flava: dose-effect curves and action spectrum determination. J Photochem Photobiol B Biol 80:78-83 